\title{
A Report of 10 Cases of Parkinson's Disease Cured by Xifengzhizhan Pills and Xifengzhizhan Capsules
}

\author{
Weifan Wang \\ TCM Internal Clinic, Xinmi, China \\ Email: 461679365@qq.com
}

How to cite this paper: Wang, W.F. (2019) A Report of 10 Cases of Parkinson's Disease Cured by Xifengzhizhan Pills and Xifengzhizhan Capsules. Advances in Parkinson's Disease, 8, 9-17. https://doi.org/10.4236/apd.2019.82002

Received: March 21, 2019

Accepted: May 17, 2019

Published: May 20, 2019

Copyright $\odot 2019$ by author(s) and Scientific Research Publishing Inc. This work is licensed under the Creative Commons Attribution International License (CC BY 4.0).

http://creativecommons.org/licenses/by/4.0/

\section{cc) (i) Open Access}

\begin{abstract}
Parkinson's Disease (PD) is a chronic progressive degeneration disease of the central nervous system of extrapyramidal system. The main pathological changes are the degeneration of the substantia nigra striatum, lack of dopamine in the brain, causing clinical performance: static tremor, muscle rigidity, slow movement and abnormal posture. It is one of the worldwide medical problems; at present, modern medicine thinks that $\mathrm{PD}$ is an incurable and permanent disease. However, in my long-term clinical work, I have cured many cases of PD patients by using Xifengzhizhan pills and Xifenzhizhan capsules, and I have found and proved that Chinese Medicine can cure PD.
\end{abstract}

\section{Keywords}

Parkinson's Disease, Chinese Medicine, Cure Parkinson's Disease, Parkinson's Disease Case Report, Xifengzhizhan Pills, Xifengzhizhan Capsules

\section{Introduction}

According to the modern medical literature data collected, at present, due to the exact pathogeny of PD is not yet clear, modern medicine mainly uses dopamine replacement therapy and dopamine receptor agonist symptomatic treatment for $\mathrm{PD}$, which can improve symptoms, but cannot cure PD. There are no reports of complete cure of PD treated with Traditional Chinese Medicine alone. I use the Traditional Chinese Medicine compound Xifengzhizhan pills and Xifengzhizhan capsules to treat Parkinson's Disease, which can not only improve the clinical symptoms, but also cure Parkinson's Disease, which is a major medical breakthrough. 


\section{Case Selection and Treatment Methods}

All treated cases were Parkinson's disease patients who had been diagnosed by experts in neurology department of senior hospitals. According to the theory of TCM, Yin governs tranquility, Yang actively, Yin governs softness, Yang governs rigidity, combined with the main clinical manifestations of Parkinson's disease, such as limb tremor and muscle stiffness, syndrome differentiation is, deficiency wind internal movement, so nourishing yin of Gan and Shen, and extinguishing wind should be the treatment principle. According to this rule, the Chinese herbal medicine with the right syndromes was selected, and the Chinese patent medicine Xifengzhichan pills and Xifengzhichan capsules were prepared. I used the Xifengzhizhan pills and Xifengzhizhan capsuless to treat PD. Both Xifengzhichao Pills and Xifengzhichan Capsules are pure Chinese herbal compound preparations. The main components of Xifengzhichan Pills are: Shudi, cornel meat, medlar, chrysanthemum, Ligustrum lucidum, Gastrodia elata, uncaria, polygonatum, asparagus, etc. The main components of Xifengzhizhan capsules are: white peony root, ejiao, turtle shell, cistanche deserticola, natural ground, radix scrophulariae, radix bidentata, etc. Xifengzhichan Pills 9 grams each time, three times a day; Xifengzhichan capsules 6 capsules each time, three times a day, oral administration.

In this article, only 10 typical cases from many cured PD patients were selected and reported as follows:

\subsection{Cases 1}

Mr. Wang is 62 years old, a farmer of Xinmi city, Henan province. From the winter of 1989, unexplained reasons, he began to appear right upper limb quiescent tremor, right upper and lower limbs and neck stiff, right leg and right foot dragging when walking. Having visited the First Affiliated Hospital and the Second Affiliated Hospital of Henan Medical College successively, he was treated by neurologists respectively, and was diagnosed PD, They prescribed Antan and levodopa to him. But, his condition continued to develop and his hands were so shaken that he could not eat and drink with his hands. Since he couldn't stand side effects of these medicines, he was forced to give up.

In July 1991, He began to take TCM Xifengzhichan Pills, 9 grams each time, and Xifengzhichan Capsules, 6 capsules each time, three times a day. Two months later, the symptoms began to improve. After one and a half years of continuous treatment, tremor, ankylosis disappeared and gait was normal in December 1992. For nearly 10 years, he was engaged in all kinds of housework and farm work as usual, pushing a car to do small business. Until the Spring Festival of 2002 , he died suddenly of hypertensive cerebral hemorrhage.

\subsection{Case 2}

Chen Xiaolei, male, 13 years old, a student from Chengou Village, Laiji Town, Xinmi City, In October 1995, for unknown reasons, his right hand trembled, his 
legs trembled, his neck stiffened, his lower limbs stiffened and his walking was unfavorable. He has been hospitalized in many hospitals in Xinmi and Zhengzhou. After being diagnosed as Parkinson's disease by experts, he has taken Antan for more than half a year. His hand trembles more and more severely, so that he can hardly carry bowls and chopsticks, can hardly write, and develops to trembling hands, stiffness of lower limbs and difficulty in walking.

On March 29, 1996, he began to take Chinese Medicine: Xifengzhichan pills 9 grams each time, three times a day; Xifengzhichan capsules 6 capsules each time, three times a day. In the second month, the tremor of both hands was from continuity to intermittence. The stiffness of neck and lower limbs began to relax. In the third month, the hands hardly trembled. The bowls and chopsticks were handled more freely. The neck and lower limbs were completely relaxed and the walking was natural. Continued treatment for three months, tremor and rigidity disappeared completely, all kinds of fine activities were completed normally, consolidation treatment for three months, he returned to normal, and returned to school in September 1996. Follow-up for 23 years showed no recurrence.

\subsection{Case 3}

Ms. Liu, 39, lives in Shengou Village, Chaohua Town, Xinmi City. In March 1996, for unknown reasons, she experienced bilateral limb quiescent tremor, upper body tilt forward, unstable walking, anxiety and insomnia. She was diagnosed as Parkinson's disease in many hospitals in Xinmi City and the provincial hospital. She took Madopa for more than half a year. Her condition continued to worsen. She could not take care of herself. She needed help in eating, dressing and walking.

On September 25, 1996, she came to see TCM, and took Xifengzhichan pills and Xifengzhichan capsules, Xifengzhichan pills 9 grams each time, three times a day; Xifengzhichan capsules 6 capsules each time, three times a day. One month later, the patient's condition tended to be stable, first of all, mental improvement and insomnia improvement; the second month tremor was greatly reduced, walking was relatively stable, eating and dressing were basically self-care; the third month, the patient's condition was further improved; after more than half a year of treatment, all kinds of symptoms completely disappeared, and the patient completely recovered. Six months after consolidation of treatment, the patient returned to work. After 22 years of follow-up, she was in good health.

\subsection{Case 4}

Mr. Yang, 72 years old, retired cadre of Wuchang District Wutie Branch Court, Wuhan City, Hubei Province. In April 1994, for unknown reasons, he suffered from resting tremor of hands and feet, stiffness of limbs, difficulty in movement, dry stool and impetuous insomnia. He had been hospitalized seven times and was diagnosed as Parkinson's disease by experts. He took western medicine, and his condition continued to worsen. By 1997, he was shaking all over, unable to 
take care of himself, only stay in bed all day uncomfortable and distressed.

On January 15, 1998, he began to take Chinese medicine Xifengzhichan Pills and Xifengzhichan Capsules, 9 grams of Xifengzhichan Pills each time, three times a day; Xifengzhichan Capsules, 6 capsules each time, three times a day. Three months later, the patient's mood was stable, his sleep improved at night, and his obstinate constipation alleviated. Six months later, the tremor began to ease, the body became softer and lighter, and the stool became clear. Nine months later, tremor and hardness were significantly reduced and he could get out of bed. One year later, the tremor basically disappeared and the patient could go downstairs to move. After one and a half years, all kinds of symptoms disappeared and recovered completely. Everyday life was like normal people. Following up for 20 years, the patient was in good health.

\subsection{Case 5}

Mr. Tong, 74 years old, retired cadre of No. 1 Middle School of Yiyang County, Henan Province. Onset in 1966, his hand fibrillation affects handwriting. The disease progressed gradually. After 1990, the hands showed continuous quiescent tremor and writing was very difficult. When eating, the hands could not hold chopsticks. When dressing, the hands could not buckle buttons and pull locks. The legs were stiff, limbs were weak and could not walk. When eating, there was no appetite, insomnia, anxiety, restlessness, and life could not take care of itself. Li Jianzhang, a famous expert in neurology department of provincial city hospital, diagnosed him with Parkinson's disease.

In July 1997, Chinese medicine Xifengzhichan Pills and Xifengzhichan Capsules were taken for treatment. Xifengzhichan Pills, 9 grams per time, three times a day, and Xifengzhichan Capsules, 6 capsules per time, three times a day. After 9 months of continuous taking, the chopsticks can be grasped during meals, and the tremor of the hands with buttons and clothes is lighter, gradually no longer tremor. By about one year, the function of the hands is basically restored. Defibrillation improved markedly, other aspects of the body also improved, appetite increased, sleep was good, walking was strong. It turned out that they could not walk a kilometer because he had little energy and stooped forward. Later, he walked three or four miles with his head held high and his chest held high. Continue to take medicine to consolidate the effect, the condition is getting better and better. Two years later, the symptoms disappeared. In the past, he had to wait for meals by others' help. In recent years, he cooked his own meals and took care of his wife with hemiplegia for many years. Until the age of 90 , he died.

\subsection{Case 6}

Mr. Wang, 72, is a primary school teacher in Changfeng Village, Xicun Town, Gongyi City, Henan Province. In 1991, he began to be tremors in both hands and head shaking. Later, the illness aggravated, which affected the work. It was 
difficult to write. It was difficult to carry bowls and chopsticks, and the movements were not flexible. The experts diagnosed him as Parkinson's disease successively in the township health center and Gongyi People's Hospital. They prescribed Antan. After taking it for a period of time, the tremor was alleviated, but it began to tremble again when he stopped taking medicine.

In November 2002, he was treated with Xifengzhichan Pills and Xifengzhichan Capsules, 9 grams of Xifengzhichan Pills each time, three times a day, and 6 capsules of Xifengzhichan Capsules each time, three times a day. For more than two months, the effect was achieved, the tremor was alleviated, and lasted for about one year. The tremor basically disappeared. As normal people, he could carry bowls and chopsticks, write with pen, consolidate treatment for half a year, and recover completely. After 16 years of follow-up, free movement, the patient remained healthy.

\subsection{Case 7}

Mr. Li, a 68-year-old man, lives in No. 8 Huanan Lane, Jianjian Road, Gongyi City. In the winter of 2002, he began to be ill, trembling hands and feet, impatient, unable to sleep at night, restless in sitting and lying, unable to eat, and unable to walk. He went to county hospitals and provincial hospitals one after another, and the experts confirmed Parkinson's disease. By the summer of 2003, the disease had developed very seriously.

He visited me in August 2003. Since then, he take TCM Xifengzhizhan pills and xifengzhizhan capsules, Xifengzhizhan pills 9 grams each time, three times a day; Xifengzhizhan capsule 6 capsules each time, three times a day. After more than two months, there were obvious effects. First of all, he was able to rest, his mood was not irritable, he was a little bit more energetic, and he was able to walk with strength. He had to rest when he walked one kilometer before taking medicine. After three months of taking the medicine, he can walk three or four kilometers before taking a rest. Then he gradually found that the hand can also hold the bowl, can also hold the chopsticks, trembling relieved. The expression of the face is also gradually different from the past, mental outlook significantly improved. After about four months, the symptoms largely disappeared. Rest, eat, walk as normal as before the illness, and he can do all housework. A full recovery followed a year of treatment. Following up to now, he has been in good health for 15 years.

\subsection{Case 8}

Ms. Ma, 70, a citizen of Luohe City, Henan Province, She began to tremble involuntarily on her right hand and right leg for unknown reasons in the spring of 1995. Her arms were stiff, her lower limbs were weak, and she was dragged by walking. To the county hospital, the director of internal medicine diagnosed Parkinson's Disease, prescribed Antan, levodopa. After taking the medicine, the symptoms were relieved, but the side effects could not be tolerated. Later, she 
went to the expert clinic in provincial hospitals. Several experts diagnosed Parkinson's disease and prescribed medicines. After taking the medicine, she felt sick in the stomach and was forced to stop using it. However, the symptoms of Parkinson's disease became more and more serious. Until the second half of 1997, the tremor of the right upper and lower limbs was persistent, the right hand could not write with a pen, and eating with chopsticks was also affected, difficulty in walking, slow movement, rigid facial expression.

On December 3, 1997, she came to visit my clinic to take Chinese medicine Xifengzhichan Pills and Xifengzhichan Capsules. After taking them for about 10 months, her condition began to improve, her facial expression improved, and her tremor stopped when he trembled. By one and a half years, the effect was very obvious. After taking the medicine for two years, all kinds of symptoms disappeared. After six months of consolidation treatment, the patient recovered completely. After 20 years of follow-up, the patient's condition remained normal.

\subsection{Case 9}

Mr. Wang, 80 years old, a cadre of Xinzheng City, Henan Province, presented with stationary tremor in his upper limbs, rigidity of muscles in his limbs and waist and back, slow movement, forward leaning in walking posture, panic in gait and disappearance of accompanying movements of his upper limbs in October 2005 for unknown reasons. He has been consulted by neurologists in Xinzheng People's Hospital, Henan People's Hospital and the First Affiliated Hospital of Zhengzhou University successively. He was diagnosed as Parkinson's disease by experts. He was treated with Madopa. After taking Madopa for two months, he stopped taking because of intolerable side effects such as nausea and vomiting. After that, the disease continued to progress, gradually from unilateral to bilateral. In the most severe cases, bilateral upper limb quiescent tremor is persistent, and all kinds of movements such as eating and dressing are restricted.

The patient came to see us on May 10, 2007. He was treated with Xifengzhichan Pills, 9 grams each time, and Xifengzhichan Capsules, 6 pills each time, three times a day. After taking Chinese medicine for 2 months, the patients began to show effects, tremor and stiffness were alleviated, movement retardation was improved, and walking posture was gradually stable. After taking it for one and a half years, it has obvious effect, tremor and hardness disappear, limbs are flexible, all kinds of activities are the same before illness, gait is normal, continue to consolidate treatment for half a year, and terminate treatment. Continue to observe for ten years, he is in good health, no Parkinson's disease symptoms return.

\subsection{Case 10}

Ms. Jiang, 78 years old, from Kuandian Town, Kuandian County, Liaoning Province, presented with right upper limb quiescent tremor for unknown reasons in May 2007. Gradual progress affected catering, limb stiffness, slow 
movement, leaning forward when walking, gait panic, frequent falls, lack of facial expression, glassy eyes, unclear speech, cough in drinking water, dry stool knot, which was diagnosed Parkinson's disease by neurologists in Kuandian County Hospital.

In October 2009, she began to take Chinese medicine Xifengzhichan pills, 9 grams each time, and Xifengzhichan capsules 6 capsules each time, three times a day. After nine months of taking the medicine, the symptoms gradually improved. About a year later, all the symptoms disappeared. After six months of consolidated treatment, the patients recovered completely and engaged in various activities and housework as normal people. After recovery, everything was normal until hemiplegia due to cerebral infarction in 2018.

\section{Discussion}

According to the viewpoint of modern medicine, if a patient is definitely diagnosed as Parkinson's disease, it cannot be cured. If treated with western medicine, only the symptoms can be improved and lifelong medication is needed. As the disease progresses, the quality of life of patients will be seriously reduced, even unable to take care of themselves. Late-stage patients may be fatal due to a variety of complications.

However, traditional Chinese medicine treatment is not the same. Although it cannot be as immediate as western medicine, most of cases need take several months to begin to produce results, but TCM can not only gradually improve clinical symptoms, but also completely cure Parkinson's disease, which is the result that western medicine cannot achieve.

The reason why Chinese medicine can cure Parkinson's disease is supposed to be the following aspects: Chinese medicine can eliminate the cause of Parkinson's disease, Although the etiology of Parkinson's disease is still unclear, from the follow-up of the cured patients, it was found that the patients had terminated treatment for many years and did not see the symptoms of Parkinson's disease again. Obviously, the etiology of Parkinson's disease has been cleared by traditional Chinese medicine. If the etiology persists, even after the treatment of traditional Chinese medicine, the symptoms will disappear temporarily and the symptoms will reappear after the termination treatment. It can also be inferred that the etiology of Parkinson's disease can be eliminated by traditional Chinese medicine. [1] [2] [3]; Chinese medicine can reverse the process of degeneration of substantia nigra in brain, The main pathological change of Parkinson's disease is substantia nigra degeneration. Modern medicine believes that this degeneration process is irreversible, and my research confirms that this degeneration process is actually reversible, that is to say, the degeneration process is not one-way, but can be reversed gradually after the etiology is cleared in the course of traditional Chinese medicine treatment. [4] [5]; Chinese medicine can repair the pathological changes of substantia nigra cells, Except for the necrotic nigral cells, the remaining pathological nigral cells can be gradually repaired under the 
action of traditional Chinese medicine until their morphology and structure are normal [6]; Substantia nigra degeneration can lead to the decrease of dopamine in the brain. After the morphology and structure of the diseased cells are gradually restored, Chinese medicine can gradually restore the function of substantia nigra to make dopamine until it reaches normal completely [7]; Chinese medicine can eventually cure all the clinical symptoms of Parkinson's disease after treatment one to two years [8]. So that patients with Parkinson's disease can recover.

Otherwise, if the cause of Parkinson's disease persists, the degeneration of the substantia nigra cannot be reversed, and the dopamine production of the substantia nigra cannot be fully restored. The symptoms of Parkinson's disease are unlikely to disappear and the disease cannot be completely cured.

In a word, the treatment of traditional Chinese medicine has a fundamental effect on Parkinson's disease, which brings hope for the recovery of Parkinson's disease patients. Parkinson's disease, a major and difficult disease in human medicine, has been conquered. The methods and drugs that can cure Parkinson's disease have been found. Parkinson's disease will no longer be an incurable lifelong disease.

\section{Conclusion}

In the past 28 years, more and more patients with Parkinson's disease have been cured by TCM. These cured cases of Parkinson's disease strongly prove that Parkinson's disease can be cured and TCM can cure Parkinson's disease.

\section{Conflicts of Interest}

The author declares no conflicts of interest regarding the publication of this paper.

\section{References}

[1] Huang, S.D., Liang, J.F. and Chen, Y.Q. (2018) Advances in Clinical and Experimental Research on the Treatment of Parkinson's Disease with Chinese Herbal Compound Prescriptions Based on Syndrome Differentiation. Chinese Journal of Traditional Chinese Medicine, 11, 52.

[2] Teng, L., Hong, F. and He, J.-C. (2018) Effect of Chinese Herbal Compound Rehmannia Prescription on FosB mRNA and Protein Expression in Rat Model of Parkinson's Disease with Dyskinesia. Chinese Journal of Traditional Chinese Medicine, 10, 4403-4406.

[3] Xue, Y. and Tuo, H.Z. (2017) Study on the Treatment and Mechanism of Parkinson's Traditional Chinese Medicine. Heilongjiang Traditional Chinese Medicine, $11,56-58$

[4] Wan, Y., Guo, C.Y. and Li, Y.M. (2017) Research Progress of Traditional Chinese Medicine in the Field of Parkinson's Disease. Journal of Neuropsychopharmacolo gy, 4, 36-42.

[5] Jiang, W.X. and Yang, X.M. (2017) Effect of Nerve Growth Liquid of Chinese Medicine on Mice with Parkinson's Disease. Chinese Journal of Traditional Chinese 
Medicine, 8, 3687-3691.

[6] Ming, J.F. and Cai, Z.L. (2017) Research Progress and Potential Value of Traditional Chinese Medicine in the Treatment of Parkinson's Disease. Journal of Practical Medicine, 12, 1904-1907.

[7] Sun, S.Z. (2017) Clinical Application of Bushen Huoxue Decoction in Parkinson's Disease. Chinese Medical Guidelines, 6, 178-179.

[8] Lang, Y.L., Xu, L.L., Kang, Z.Z., Yang, L. and Zhang, L.P. (2016) Research Progress of Chinese Herbal Medicine against Parkinson's Disease. Zhejiang Journal of Integrated Traditional Chinese and Western Medicine, 12, 1143-1146. 